\title{
Macht und Ohnmacht der Medien in Demokratisierungsprozessen: Lehren aus dem „Arabischen Frühling“6
}

Der Einfluss der Medien auf Demokratisierungsprozesse ist von der Wissenschaft lange ignoriert worden. Während die Medien- und Kommunikationswissenschaft sich nahezu vollständig auf die Erfahrungswelt der entwickelten Demokratien in Europa und Nordamerika stützte und Forschungen zu Asien, Afrika und Lateinamerika selten waren, konzentrierte sich die Politikwissenschaft auf Facetten ,realistischer" Schulen, wobei nicht Kommunikation und Wahrnehmungen, sondern politische und ökonomische Interessen als treibende Kräfte der weltweiten Entwicklung zur Demokratie galten. Dies hatte vor allem mit Erfahrungswerten zu tun. Die Massenmedien hatten sich bis in die neunziger Jahre hinein nicht gerade als Avantgarde von Demokratisierungsprozessen hervorgetan. Während Künstler und Intellektuelle beispielsweise als Dissidenten dazu beitrugen, den von der Sowjetunion beherrschten Ostblock zu Fall zu bringen, waren dessen Medien zu leicht propagandistisch beherrschbar. Der moderne Journalismus trägt zwar Züge einer freien Profession, er ist aber vor allem ein industrieller Komplex, der von der Rotationspresse bis zum Funkhaus auf erhebliche Ressourcen angewiesen ist, die von jedem autoritären System leicht kontrolliert werden können.

Das Ignorieren der Medien hatte aber auch etwas mit paradigmatischen Fixierungen der Politikwissenschaft zu tun und der langjährigen Unfähigkeit, „,Kommunikation" als eine der bedeutsamen politischen Ressourcen politischer Entwicklung ernst zu nehmen. Erst bahnbrechende Arbeiten wie der „Strukturwandel der Öffentlichkeit“" oder die „Theorie des Kommunikativen Handelns“" von Jürgen Habermas veränderten den Blick für die Bedeutsamkeit der ,politischen Kommunikation“ (Habermas 1990, 1995). Hinzu kam im Kalten Krieg die langsame Aufwertung perzeptionstheoretischer Ansätze, die Feindbilder auf beiden Seiten untersuchten (MccGwire 1987). Und nach dem Ende des Ost-West-Konflikts kam ein dritter Aspekt hinzu: rapide technische Entwicklungen der Medien wie das direkt empfangbare Satellitenfernsehen und die Entstehung des Internets erwiesen sich als Unsicherheitsfaktoren der Politik. Die Vorherrschaft der traditionellen Gremiendemokratien in Europa und Nordamerika, die nun zu „Mediendemokratien“ zu werden schienen (Donsbach/Jandura 2003), wurde dadurch ebenso in Frage gestellt wie das 
Propagandamonopol autoritärer Staatsführungen, die zunehmend von innerhalb wie außerhalb ihrer Staaten medialem Druck ausgesetzt waren.

Alle Entwicklungen zusammen führten nicht nur in der Wissenschaft, sondern auch in der breiten Öffentlichkeit zu einer wachsenden Offenheit für die Wirkung der Medien auf Demokratie und Demokratisierung, die nun allerdings in paradoxer Weise bald überschätzt wurde. Aus der Ignoranz wurde vielfach eine technizistische Euphorie. Wo ein Systemwandel mit traditioneller Machtpolitik nicht zu machen war und sich die Bevölkerungen in Aufständen wehrte, war schnell von „FacebookRevolutionen“ die Rede - so geschehen etwa bei der „grünen Revolution“ in Iran 2009 oder während des Arabischen Frühlings in Tunesien und Ägypten 2010/11. Beide Demokratisierungsprozesse sind jedoch nach anfänglich großen Hoffnungen bislang gescheitert, was erneut die Frage aufwirft: Welchen Einfluss üben Medien in Demokratisierungsprozessen denn nun wirklich aus?

Parick J. McConnell und Lee B. Becker haben die konträren Sichtweisen der Wissenschaft auf Macht und Ohnmacht der Medien in Demokratisierungsprozessen in sechs „Schulen“ zusammengefasst, die sich in zwei große Richtungen gliedern lassen, wovon die eine die Überlegenheit der Medien vor anderen Kräften postuliert („media-supremist position“), während die andere das Gegenteil behauptet („,emocracy-supremist position“) (McConnell/Becker 2002: 12 ff.). Man würde es sich allerdings zu leicht machen, wenn man die Frage des Zusammenhangs zwischen Medien und Transformation einfach als ungeklärt und offen bezeichnen würde. Gerade der Arabische Frühling war ein Lehrbeispiel dafür, wie Medien und Öffentlichkeit unter bestimmten Bedingungen ein Machtfaktor sein können, während in anderen Situationen die Statusfaktoren Macht und Kapital dominieren.

In einer Art vorweggenommener Konklusion lässt sich sagen: eine von der $\mathrm{Zi}$ vilgesellschaft und weniger von herrschenden Eliten ausgehende Demokratisierung muss sich der Möglichkeiten der Medien sehr bewusst und strategisch bedienen, darf sich aber nicht auf diese allein verlassen und sollte traditionelle Politikformen der parlamentarischen Demokratie nicht vernachlässigen. Ein Paradigmenwechsel zum Medienzentrismus in der zivilgesellschaftlichen Forschung wäre eine Hypertrophierung der Einschätzung von Medienmacht und eine Idealisierung post-moderner und post-demokratischer Handlungsformen von sozialen Bewegungen. Realistisch hingegen ist ein moderater Standpunkt, der die Wechselwirkungen zwischen kommunikativer Vernetzung und Systemmacht an den Knotenpunkten medialer Netze ins Auge fasst. 


\section{Autoritäre Phase: Arabische Medien als „Ersatz“ für politische Parteien}

Die Vorstellung von einer Unvereinbarkeit von Demokratie und Islam bzw. islamischer Welt hat sich in der Politikwissenschaft lange behaupten können (Bromley 1997). Zwar war schon vor dem Arabischen Frühling bekannt, dass in den überwiegend autoritären Staaten Nordafrikas sowie des Nahen und Mittleren Ostens zahlreiche säkulare wie islamistische zivilgesellschaftliche Kräfte existierten und eine politische Kultur, die mehrheitlich auf Demokratisierung drängte (Esposito/ Mogahed 2007). Dabei wirkte die Tatsache, dass anders als im Westen Religion eine bedeutsame Rolle für eine oft radikale Opposition spielte, auf viele Beobachter verstörend. Dennoch wurden sowohl das friedliche Szenario einer zwischen radikalen Kräften zu verhandelnden Demokratisierung als auch das Abgleiten in Bürgerkriege im Zuge einer Transformation als zwei mögliche Entwicklungswege erkannt (Hafez 2010: 2). Dominierend aber war in der Wissenschaft die Auffassung, zivilgesellschaftliche Kräfte seien eben zu schwach, um den autoritären Staat zu gefährden (Schlumberger 2008). Der Arabische Frühling wurde ebenso wenig wie seinerzeit der Fall der Sowjetunion vorausgesehen.

Bereits vor dem Arabischen Frühling gab es allerdings einen Hoffnungsschimmer: Die Emergenz einer arabischen Medienöffentlichkeit, die eng mit dem Namen des 1996 gegründeten qatarischen Fernsehsenders Al-Jazeera verbunden war und sich in einer Vielzahl arabischer Fernsehkanäle ausdrückte, die in der arabischen Welt grenzüberschreitend empfangbar waren, führte zu überraschenden Entwicklungen. Das spezifische Verständnis Al-Jazeeras, als Sprachrohr der unterdrückten arabischen Bevölkerungen zu fungieren, neue Formen des Polit-Talks, Call-In-Sendungen usw. führten nicht nur zu zahlreichen Tabubrüchen durch einen kritischen Umgang mit arabischen Potentaten und sozialen Fehlentwicklungen, sondern förderten in der Region bereits vor demokratischen Systemwechseln die Entstehung einer teilweise freien Öffentlichkeit (Eickelman/Anderson 1999). Viele arabische Autokratien von Marokko über Ägypten bis Jordanien mussten sich den Verhältnissen beugen, sie duldeten den Wildwuchs der Satellitenantennen. Es entstanden liberale Autokratien mit begrenzter Meinungsfreiheit.

Bald setzte ein Nachdenken darüber ein, ob die Medien eine Art „Ersatz“ für die oft fehlenden politischen Oppositionsparteien seien und die Demokratisierung fördern könnten (Hafez 2006). Schon damals allerdings bestanden theoretische Unsicherheiten. Was konnten die Medien alleine bewirken? Schließlich waren sie selbst kein primärer sozialer Akteur. Wie weit waren sie in der Lage, die Herausbildung ideologischer Alternativen und die Mobilisierung einer Protestbewegung, die in der Transformationslehre als Merkmal der Konfliktfähigkeit der Opposition genannt 
wurden, herzustellen? Trotz aller Begeisterung über die neuen Medien war schließlich in der arabischen Welt keinerlei Systemwandel in Sicht, die Medienrevolution blieb folgenlos, was auf eher sporadische Bindungen zur Zivilgesellschaft und allenfalls langfristige positive Wirkungen auf die politischen Kulturen schließen ließ, die keine direkten Konsequenzen für die politischen Systeme hatten (Hafez 2008 a, 2008 b, Schäfer 2009). Dennoch war das Thema einer „kommunikativen Wende“ mit möglicherweise revolutionären Auswirkungen bereits vor dem Arabischen Frühling auf der Agenda. ${ }^{1}$

\section{Transition: der Arabische Frühling als Medienrevolution?}

Demokratisierungsprozesse werden klassischerweise in drei Phasen eingeteilt: Autoritarismus, Transition und Konsolidierung. Während die Transition mit der weithin sichtbaren Verunsicherung autoritärer Herrschaft einsetzt, kann nach der Abhaltung erster freier Wahlen die Konsolidierung der Demokratie durch den Umbau der Institutionen beginnen. An der Einleitung der Transition in Tunesien und Ägypten waren maßgeblich die neuen sozialen Medien des Internets beteiligt. Mehr noch als in Tunesien war Ägypten das Zentrum der arabischen Bloggerbewegung. Bereits während der Iranischen Revolution 1978/79 waren kleine Medien wie Videokassetten des Revolutionsführers Khomeini massenhaft ins Land geschmuggelt worden. Die neuen interaktiven Medien der Jahre 2010/11 besaßen den Vorteil, nicht nur die Artikulation und Mobilisierung, sondern auch die Organisation der Opposition zu fördern, was für die Durchführung eines in diesem Fall spontanen Aufstandes wichtig war. Der Kreis schien sich zu schließen: was Massenmedien wie Al-Jazeera noch gefehlt hatte - die direkte Bindung zur Basis - ermöglichte das Internet. In der Sprache der systemtheoretischen Öffentlichkeitstheorie waren nun alle Ebenen beteiligt: die private Encounter-Öffentlichkeit ebenso wie die Versammlungskommunikation (virtuell im Internet) und die Massenmedien (Al-Jazeera berichtet 24 Stunden live aus Ägypten) (Neidhardt 1994). Technisch verstärkt baute sich eine Welle des Protestes auf, die bewirkte, was in der arabischen Welt Jahrzehnte lang immer wieder in Protesten versucht worden war: scheinbar unerschütterliche autoritäre Regimes wurden binnen kürzester Zeit beseitigt. Die Macht der Medien schien ein für alle Mal demonstriert.

Auch wenn man die anschiebende Wirkung der sozialen Medien gerade in den ersten Tagen der ägyptischen Aufstände anerkennt, war der Arabische Frühling

1 Communicative Turn - Arab Media and the Culture of Democracy, Keynote von Kai Hafez, $14^{\text {th }}$ Annual Conference, The Arab-U.S. Association for Communication Educators, American University Cairo, Kairo, 7. November 2009. 
weder eine „Facebook-Revolution“ noch eine Medienrevolution (Lynch 2011). Nicht nur wurde das Internet nach wenigen Tagen in Ägypten abgeschaltet, was die Aufstände dennoch nicht beendete. Klassische Massenmedien wie Al-Jazeera, das die Ereignisse in Tunesien fast verschlafen hatte und über Bahrein auf Druck der qatarischen Regierung nicht senden durfte, machten die Aufstände in Ägypten zu einem Großereignis und rückten sich selbst einmal mehr ins Zentrum der Weltbeachtung. Ein zweiter Grund ist, dass mündliche soziale Kommunikation sowie traditionelle Formen der politischen Kommunikation wie Flugblätter, Graffities, also uralte Kulturtechniken, weitaus wirksamer waren als die neuen elektronischen Medien, obwohl sie in der Analyse oft vergessen werden. Die meisten Protestierenden waren nie im Internet oder bei Facebook aktiv geworden und schon gar nicht bei „Twitter", das in den Aufständen ohnehin noch keine Rolle spielte. Demokratische Aufstände hatte es in Lateinamerika und Osteuropa schon lange vor dem Zeitalter der digitalen Medien gegeben, und der Arabische Frühling war bestenfalls ein Beispiel für den sogenannten multi-step flow of communication, das Zusammenspiel von Medien, sozialen Meinungsführern und Sympathisanten. Internet und Satellitenfernsehen waren ein wichtiger Teil einer transitorischen Gemengelage, die allerdings über Mund-zu-Mund-Propaganda eine ebenso große Dynamik entfaltete.

\section{Konsolidierung: das Scheitern der „Mediendemokratie“}

Ebenso wenig wie die Erfolge der Transition gingen die Misserfolge der Konsolidierung - besonders in Ägypten, das seit dem Staatscoup von 2013 de facto wieder eine Autokratie ist - vollständig auf das Konto der Medien. Die Zivilgesellschaften hatten sich der Medien und anderer Kommunikationsformen bedient, um die Hauptfunktionen einer Opposition, die Artikulation politischer Eliten und eine konfliktfähige Organisation und Mobilisierung der Bevölkerung, zu erreichen und eine kurzfristige soziale Bewegung über Lagergrenzen hinweg zu bilden. Sowohl die öffentliche Artikulation des Demokratieprojekts als auch die Konfliktfähigkeit der Opposition haben sich aber zumindest in Ägypten als neuralgische Punkte der Konsolidierung erwiesen. Mit den demokratischen Wahlen zerfiel nicht nur die Einheit der antiautoritären Bewegung, sondern es setzten Zerfallserscheinungen auf allen Ebenen ein, was nicht hauptsächlich an den Medien lag, von diesen aber mitverschuldet wurde.

Was den ersten Punkt der Artikulation betrifft, ist insbesondere die junge ägyptische Demokratie in den Jahren 2011-2013 hinter dem Habermas'schen Ideal einer rationalen Deliberation zurückgeblieben. Charakteristisch war vielmehr eine radikale Polarisierung der Öffentlichkeit mit einer dominanten Trennung zwischen sä- 
kularen und islamistischen Lagern. Diejenigen Medien, die zu dem den Muslimbrüdern nahe stehenden Präsidenten Mursi tendierten, zum Beispiel die Fernsehsender Masr 25, al-Rahma, al-Hafez, al-Nas oder al-Khalijiya, waren sehr einseitig in ihrer Berichterstattung. Sie verbreiteten vielfach Gerüchte über Gegner Mursis und über seine Verfassung, bezeichneten sie als Trunkenbolde und soziale Parasiten. Die islamistischen Medien hingegen verhielten sich oft sektiererisch und propagandistisch. Viele Nutzer bekamen Angst vor einer nachhaltigen Islamisierung des Landes, zumal ja auch die staatlichen Medien von Mursi kontrolliert wurden. Allerdings war auch das Auftreten der gegen Mursi gerichteten Medien vielfach radikal zu nennen. Die linksliberale Zeitung Al-Tahrir beispielsweise war sehr pauschal gegen die Muslimbrüderschaft eingestellt: Eine Tatsache, die selbst von Journalisten innerhalb des Blattes kritisiert wurde, die sich mehr innere Meinungsfreiheit gewünscht hätten. ${ }^{2}$ Nur sehr wenige Medien wie die Tageszeitung Al-Masry AlYoum konnten für sich beanspruchen, zentristisch und zumindest vergleichsweise unabhängig zu berichten.

Das Mediensystem der ersten ägyptischen Demokratie seit dem Nasseristischen Militärputsch von 1952 war ,pluralistisch“ in dem Sinne, dass es alle möglichen politischen Anschauungen zum Ausdruck brachte. Es war aber auch „radikal“, weil es vielfach eine politische Kultur transportierte, die die Existenzberechtigung des politischen Gegners leugnete. Auch in erfolgreichen demokratischen Mediensystemen müssen Medien nicht überparteilich sein. Im südeuropäischen Mittelmeerraum etwa weisen viele Medien noch immer enge Bindungen zu politischen Parteien aus (Hallin/Mancini 2004). In Ägypten aber überschritten die Medien vielfach das Maß eines liberalen Grundkonsenses - ein Vergleich mit der Weimarer Republik ist hier durchaus angebracht. Doch während in Deutschland politische Richtungen noch immer erkennbar sind, hat seit dem Zweiten Weltkrieg ein liberaler Grundkonsens Einzug gehalten, der ungeachtet aller politischer Differenzen die Stabilität der Demokratie absichert - eine entscheidender Schritt, der in Ägypten noch fehlt.

Die Ursachen für die radikale Polarisierung liegen in gesellschaftlichen Strukturen, nicht so sehr in den Medien - allerdings werden sie durch Eigenlogiken der Medien verstärkt. Das ägyptische Mediensystem während des Arabischen Frühlings war durch eine starke Tendenz der Boulevardisierung und des „Politainment“ gekennzeichnet, was beispielsweise in der Skandalisierung des Nobelpreisträgers Mohammed el-Baradei und seiner Tochter zum Ausdruck kam. Nach Jahrzehnten der autoritären Zensur genossen viele Menschen ihre neu gewonnene grenzenlose Mei-

2 Für diese Information danke ich meiner Doktorandin, Nadia Leihs, die in Ägypten zahlreiche Interviews mit Journalisten durchgeführt hat. 
nungsfreiheit. Ein weiterer Aspekt kam hinzu: der starke Einfluss der Besitzstrukturen und des Medienkapitals auf die jeweilige politische Richtung des Mediums. Nicht nur, dass ein großer Teil des Medienkapitals noch immer in den Händen der alten Eliten lag (Sakr 2013), die als „,beharrende Gewalten“ hinter den Kulissen an einer Gegenrevolution arbeiteten und ihre Zeitungen und Fernsehsender zu extremer Kritik der Regierung Mursi einsetzten. Die ausbleibende Reform des großen staatlichen Mediensektors, der von der Regierung Mursi gleichgeschaltet wurde, verhinderte jede Form der ethischen Selbstkontrolle, Unabhängigkeit und inneren redaktionellen Meinungsfreiheit. Die finanzielle Abhängigkeit der meisten ägyptischen Journalisten schließlich machte sie anfällig für politische Interventionen.

In der Kommunikationswissenschaft hat man sich heute von einer Perspektive einer einseitigen manipulativen Medienwirkung zu Recht abgewandt. Populistische Meinungsmache ist nicht nur deswegen erfolgreich, weil sie von Medien verbreitet wird, sondern auch, weil sie Anhänger findet, was uns daran erinnert, dass Kommunikation eben nur eine von mehreren politischen Ressourcen ist. Massenmedien wie auch soziale Medien sind an sich wenig geeignet, Demokratisierung zu fördern, solange die gesellschaftlichen Konflikte einer stark aufgeheizten Klassengesellschaft dies verhindern. Ägypter haben sich Jahrzehnte lang hinter Gruppenzugehörigkeiten, in politischen Lagern und Netzwerken verschanzt, weil diese den in Wirklichkeit schwachen Staat, der nicht in der Lage war, seine sozialen Aufgaben zu erfüllen, ersetzen mussten. Diese Geschichte hat nun paradoxerweise den Wiederaufbau eines demokratischen Staatswesens behindert. So erstaunlich reif die arabische politische Kultur sich während des antiautoritären Arabischen Frühlings zeigte, so gering ausgeprägt ist die soziale Solidarität jenseits der eigenen Gruppenzugehörigkeit.

Hier nun gelangen wir zum zweiten Bereich, der hohe Erwartungen an die Demokratieförderung durch Medien geweckt hatte: die Mobilisierung und Organisation der Bevölkerung. Es ist eigentlich eine Binsenweisheit, muss aber dennoch betont werden: eine vernünftige Demokratie wird es in Ägypten ohne starke Vertretung aller gesellschaftlichen Kräfte durch politische Parteien nicht geben. Wenngleich manche Medien in autoritären Zeiten vor allem grenzüberschreitend als „Ersatz" politischer Parteien fungieren können, weil die Regimes Parteiarbeit verhindern, endet dieses Mandat doch spätestens mit den ersten freien Wahlen. Was wie ein Widerspruch klingt, aus dem Lagerdenken herauskommen und gleichzeitig Parteien fördern zu wollen, ist in Wirklichkeit keiner. Nicht nur ist es die Aufgabe von Parteien, die politische Meinungsbildung im Sinne eines moderaten Meinungspluralismus programmatisch zu fördern und so die radikale Polarisierung zu transformieren. Sie sind auch der Dreh- und Angelpunkt einer Kontrolle der Staatsmacht. 
In Ägypten hat sich gezeigt, dass die Transitions- und Konfliktfähigkeit der Medien im Zusammengehen mit einer Protestbewegung zwar kurzfristig erstaunlich groß, langfristig aber denkbar begrenzt ist. Eines der größten Probleme der ägyptischen Konsolidierung war die Unfähigkeit gerade der säkularen Parteien zur Selbstorganisation. Die Partei des Nobelpreisträgers Mohammed al-Baradai etwa war berühmt dafür, am Abend vor Parteiversammlungen willkürlich Sympathisanten anzurufen, um die Hallen zu füllen: ordentliche Mitgliederstrukturen gab es nicht. In dieser Form hatten die säkularen Kräfte keine Chance gegen die bei Wahlen gut mobilisierenden und daher auch siegreichen Islamisten. Die Dauermobilisierung einer außerparlamentarischen Opposition auf dem Tahrir-Platz in Kairo war eher eine Verlegenheitslösung, die öffentliche Unruhe schürte und dem Militär eine Handhabe gab, die Demokratie im Staatscoup vom Juli 2013 wieder abzuschaffen.

Man muss ohne Zweifel anerkennen, dass ein Bündnis zwischen Medien und sozialen Bewegungen nicht ausreicht, um in allen Phasen der Demokratisierung wirkungsvoll zu bleiben. Solange die Demokratisierung nur aus einer medialen Verstärkung von Protesten besteht und ordentliche parlamentarische Arbeit ausbleibt, ist nicht nur eine populistische Aufheizung der Öffentlichkeit zu erwarten, sondern dem politischen System fehlt jede interne Balance, um über die Gewaltenteilung neo-autoritäre Bestrebungen zu verhindern. Dass die Arbeiterschaft einen erheblichen Anteil am Arabischen Frühling in Ägypten hatte, wird in der Wissenschaft mit Nachdruck betont - nur leider war deren Durchsetzungskraft zu schwach.

\section{Fazit}

Am Ende liefert der Arabische Frühling die Erkenntnis, dass Medien eine durchaus stimulierende Wirkung auf die politische Kultur ausüben können, weil sie die Bereitschaft zur Meinungsfreiheit fördern und gar zur Formierung von Protestbewegungen beitragen können. Sie sind aber nur sehr eingeschränkt in der Lage, Funktionen klassischer politischer Akteure zu übernehmen und können vorhandene Schwächen der zivilgesellschaftlichen Kultur und der organisierten Konfliktfähigkeit nicht dauerhaft kompensieren. Geht man von der Unterteilung der Transformation in drei Phasen aus, dann ist die Medienwirkung in der autoritären Phase dort am stärksten, wo liberale Autokratien den Medien eine wie immer auch begrenzte Entfaltungsmöglichkeit bieten. Aus dieser im Nachhinein für das System gefährlichen Liberalität der Regimes Mubarak hat das Regime al-Sisi gelernt und unterbindet heute, ähnlich wie in der Zeit Nassers, wieder jede politische Kritik in Ägypten. Medienmacht kann also nicht nur die Demokratie fördern, sondern auch Rückentwicklungen zum harten Autoritarismus bewirken. 
Medien wirken demokratiefördernd auch in der Phase der Transition, die Vielzahl nationaler und transnationaler Medien machte es heute für autoritäre Regimes fast unmöglich, in Zeiten des Aufruhrs vollständige Informationskontrolle herzustellen. Von dem Zeitpunkt an allerdings, wo nach Wahlen das Konsolidierungswerk beginnt, werden gerade die großen Massenmedien für das nationale Aufbauwerk und die Konsensfindung in der neuen Demokratie wichtiger - und damit werden die Medien erneut anfälliger für strukturelle Interventionen von Macht und Kapital. Da eine dauerhafte außerparlamentarische Demokratie kein gangbarer Weg für ein modernes Staatswesen ist, sind auch die sozialen Medien des Internets oder andere kleine Medien nicht der entscheidende Ort der Konsolidierungsöffentlichkeit. Die Problematik junger Demokratien, die sich aus der rapiden strukturellen Überforderung der Massenmedien ergibt, haben auch andere Länder wie Indonesien nach 1998 erlebt, auch wenn die Demokratie dort stabiler war, weil die politische Landschaft bei allen Konflikten auch eine Tradition der Konsensbereitschaft mitbrachte.

Insgesamt scheint ein radikaler Paradigmenwechsel, der Medien und Kommunikation einen Primat vor Macht und Kapital einräumt, in Prozessen der Demokratisierung trotz aller beeindruckenden Medienwirkung nicht erforderlich zu sein. Die demokratisierungsfördernde Macht der Medien ist sichtbar begrenzt. Dennoch wäre es falsch, wenn sich die Politikwissenschaft in ihrer langjährigen Ignoranz des Faktors „Medien“ im Prozess demokratischer Transformationen bestätigt fühlen würde. Es gibt wenig Grund, sich vollständig auf die Seite der democracy-supremist position zu schlagen, sondern ein differenzierter Standpunkt muss gefunden werden. In einem moderaten Netzwerkansatz wie dem von Jan van Dijk sind, anders als im berühmten Werk „Informationsgesellschaft“ von Manuel Castells, nicht die Netzwerke der Medien die Grundlage der modernen Gesellschaft, sondern Individuen, Gruppen, Organisationen, kurz: die traditionellen Akteure der Politik- und Sozialtheorie bleiben die Grundeinheiten jeder Theoriebildung, auch wenn sie sich zunehmend miteinander verbinden (van Dijk 2012: 33). So ähnlich muss auch der Einfluss von Medien auf Demokratisierungen bilanziert werden: Ihre Fähigkeit, kurzfristig durch Vernetzung neue Akteure wie soziale Bewegungen und Protestbewegungen zu katalysieren, ist erstaunlich. Ihre langfristige Ohnmacht im Angesicht der nicht-mediatisierten Macht und des Kapitals ist allerdings ebenso atemberaubend. 


\section{Literatur}

Bromley, Simon, 1997: Middle East Exceptionalism - Myth or Reality?, in: David Potter/David Goldblatt/Margaret Kiloh/Paul Lewis (Hrsg.), Democratization, Cambridge, 321-344.

Donsbach, Wolfgang/Jandura, Olaf (Hrsg.), 2003: Chancen und Gefahren der Mediendemokratie, Konstanz.

Eickelman, Dale F./Anderson, Jon W., 1999: New Media in the Muslim World. The Emerging Public Sphere, Bloomington/Indianapolis.

Esposito, John L./Mogahed, Dalia, 2007: Who Speaks for Islam? What a Billion Muslims really think. Based on Galup's World Poll - the Largest Study of its Kind, New York.

Habermas, Jürgen, 1990: Strukturwandel der Öffentlichkeit, Frankfurt (zuerst 1962).

Habermas, Jürgen, 1995: Theorie des kommunikativen Handelns, 2. Bde, 4., durchges. Aufl., Frankfurt.

Hafez, Kai, 2006: Arab Satellite Broadcasting: Democracy without Political Parties, in: Transnational Broadcasting Studies 1/2, 275-297.

Hafez, Kai, 2008 a: The Role of Media in the Arab World's Transformation Process, in: Christian-Peter Hanelt/Almut Möller (Hrsg.), Bound to Cooperate: Europe and the Middle East II, Gütersloh, 321-339

Hafez, Kai, 2008 b: Introduction: Arab Media - Power and Weakness, in: Kai Hafez (Hrsg.), Arab Media - Power and Weakness, London/New York, 1-16

Hafez, Kai, 2010: Radicalism and Political Reform in the Islamic and Western Worlds, Cambridge.

Hallin, Daniel C./Mancini, Paolo, 2004: Comparing Media Systems: Three Models of Media and Politics, Cambridge.

Lynch, Marc, 2011: After Egypt: The Limits and the Promise of Online Challenges to the Authoritarian Arab State, in: Perspectives on Politics 9/2, 301-310.

McConnell, Patrick/Becker, Lee B., 2002: The Role of the Media in Democratization. Paper Presented to the Political Communication Section of the International Association for Media and Communication Research at the Barcelona Conference, July 2002, http://www.grady.uga.edu/coxcenter/activities/Act 2001_to_2002/Materials01-02/DemocratizationIAMCRJuly2002.pdf (Stand: 29.10.2014).

MccGwire, Michael, 1987: The Genesis of Soviet Threat Perception, Edited by The Brookings Institution, Washington D.C. 
Neidhardt, Friedhelm, 1994: Öffentlichkeit, öffentliche Meinung, soziale Bewegungen, in: ders. (Hrsg.), Öffentlichkeit, öffentliche Meinung, soziale Bewegungen (Sonderheft der Kölner Zeitschrift für Soziologie und Sozialpsychologie), Opladen, 7-41.

Sakr, Naomi, 2013: Transformations in Egyptian Journalism, London.

Schäfer, Michael, 2009: Protest in Ägypten. Wie Al-Jazeera und andere Medien die Kifaya-Bewegung möglich machten, Berlin.

Schlumberger, Oliver, 2008: Autoritarismus in der araischen Welt. Ursachen, Trends und internationale Demokratieförderung, Baden-Baden.

van Dijk, Jan, 2012: The Network Society, London u. a. (3. Aufl.).

Korrespondenzanschrift:

Prof. Dr. Kai Hafez

Universität Erfurt

Philosophische Fakultät

Seminar für Medien- und Kommunikationswissenschaft

Nordhäuser Str. 63

99089 Erfurt

E-Mail: kai.hafez@uni-erfurt.de 